
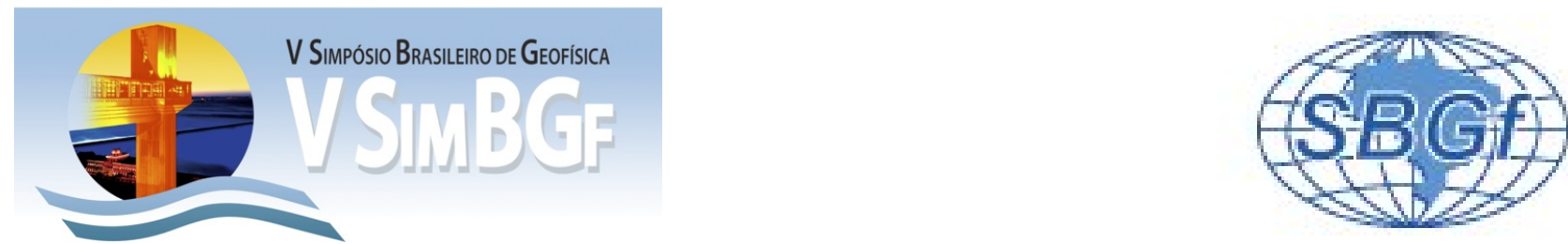

\title{
Filtragem de ruído tipo swell-noise aplicando o método de decomposição em modos empíricos
}

Antônio Jorge Sá e Milton J. Porsani, LAGEP/CPGG/IGEO/UFBA e INCT-GP/CNPq

Copyright 2012, SBGf - Sociedade Brasileira de Geofísica Este texto foi preparado para a apresentação no $V$ Simpósio Brasileiro de Geofísica, Salvador, 27 a 29 de novembro de 2012. Seu conteúdo foi revisado pelo Comitê Técnico do V SimBGf, mas não necessariamente representa a opinião da SBGf ou de seus associados. É proibida a reprodução total ou parcial deste material para propósitos comerciais sem prévia autorização da SBGf.

\section{ABSTRACT}

Generally, the removal and attenuation of noise from a seismic data is performed using methods based on the one-dimensional and two-dimensional Fourier transform. In this study we tested the method of Empirical Mode Decomposition (EMD) for removal and mitigation of noise type swell noise, present in marine seismic reflection data. The method DME decomposes adaptively, from frequency high to low, a signal is not stationary in simple oscillatory components, which are the Intrinsic Mode Functions (IMFs). Each IMF has a frequency band characteristic and the filtering can be done by subtracting one or more IMFs of the original data. The DME method is easy computational implementation, work trace-to-trace and has relatively low computational cost. The method DME was applied to a family of traces, of shot point common from a real marine seismic dataand proved to be very effective in removing this type of noise. To filter the noise type swell noise the data was broken down and filtered in $6^{\circ}$ IMF, the IMFs greater than 6 were subtracted from the original data.

\section{INTRODUÇÃO}

O dado sísmico registrado é composto pelo sinal que por sua vez é sobreposto pelos ruídos. Entende-se como sinal as reflexões primárias e as difrações e como ruído qualquer outro tipo de evento. Em um dado sísmico de reflexão, quanto maior a razão sinal-ruído (S/R), meIhor é a qualidade. A razão $S / R$ descreve a força do sinal relativa a força do ruído, isto é, a razão entre o pico de amplitude do sinal e a média máxima da amplitude do ruído em uma janela de tempo. Em uma aquisição sísmica marítima convencional, um dos ruídos aleatórios mais comum é o swell noise. Este ruído é gerado pelo movimento das ondas do mar, quando a aquisição sísmica é realizada em condição de mar re- volto. É caracterizado por possuir amplitude muito alta e baixa frequência, geralmente variando de 2 até $10 \mathrm{~Hz}$, podendo chegar até $15 \mathrm{~Hz}$ (Elboth e Hermansen, 2009). Este ruído aparece como uma faixa vertical ao longo do traço. Uma modo simples de remover este ruído é aplicar um filtro de frequência, tipo corta baixa ou passa alta, porém este filtro reduz a banda de frequência, eliminando as componentes de baixa frequência. Outros métodos utilizados são: (i) o filtro preditivo $F-X$, que funciona bem para remoção de alguns tipos de ruídos incoerentes, contudo não preserva a amplitude e (ii) o filtro tempo-frequência, que segundo (Elboth et al., 2009) é um filtro efetivo, que preserva a amplitude do sinal, além de ser uma ferramenta robusta que gera um resultado superior comparado com os métodos convencionais.

O método DME, é uma técnica para análise de sinais não-estacionários e não-lineares, que foi desenvolvido pelos pesquisadores da NASA, como uma ferramenta alternativa à transformada de Fourier para análise de dados uni-dimensionais. É um método adaptativo e muito eficiente, baseado na extração direta da energia associada com as várias escalas de tempo intrínseco, que são os parâmetros mais importante do sistema, expressa em Funções de Modos Intrínsecos (FMls) (Huang e Norden, 1998).

O método DME a partir do trabalho de (Huang e Norden, 1998) foi objeto de vários estudos, com diversas finalidades: (Flandrin et al., 2004) estudando do DME, chegaram a conclusão que o método DME atua de forma semelhante a bank filter. (Meignen e Perrier, 2007) introduziu uma nova abordagem para o DME, baseado na construção do envelope médio através da resolução de um problema de programação quadrática com restrições de igualdade e desigualdade. (Bekara e Van der Baan, 2009) e (Zegadi et al., 2009) trabalharam com método DME para atenuação de ruídos coerentes e aleatórios. (Ferreira, 2010) e (Ferreira et al., 2010) trabalharam com o método de DME para atenuação de ruído de rolamento em dado sísmico. (Alves et al., 2011) trabaIharam com método de DME em conjunto com a transformada radial (TR) para filtragem de eventos lineares.

\section{MÉTODO DE DECOMPOSIÇÃO EM MODOS EMPÍRICOS (DME)}

Algumas características importantes distingue o método 
DME de outros métodos de decomposição, tais como Fourier ou transformada wavelet. Este último decompõe o sinal usando uma base pre-definida, por exemplo, senos e cossenos ou uma wavelet mãe. Já o método DME não utiliza nenhuma base a priori uma vez que ela é derivada adaptativamente diretamente dos dados. Além disso, o método DME utiliza interpolações spline, sendo portanto menos afetado pelo espaçamento de amostragem irregular, em contraste com a transformada Fourier, que normalmente requerem intervalo de amostragem regular (Bekara e Van der Baan, 2009).

O método DME decompõe o sinal independentemente das características de fase ou de propriedade estatísticas que variam ao longo do traço (Ferreira, 2010). O princípio desta técnica é decompor adaptativamente um dado sinal $x(t)$ em componentes oscilatórias FMls, obtidas a partir do sinal, por um processo chamado separação. $\mathrm{O}$ algoritmo identifica as FMls por meio de escalas de tempo características, definidas localmente pelo lapso de tempo entre dois extremos de um modo oscilatório ou pelo lapso de tempo entre dois cruzamentos de zero do modo oscilatório (Zegadi et al., 2009).

A decomposição é baseada nos seguintes pressupostos: (Huang e Norden, 1998)

1. O sinal deve ter pelo menos dois extremos, sendo um máximo e um mínimo;

2. A escala de tempo característica é definida pelo lapso de tempo entre os extremos;

3. Se o dado sísmico for totalmente desprovido de extremos, mas possui apenas os pontos de inflexão, então ele pode ser diferenciado uma ou mais vezes para encontrar os extremos. Os resultados finais podem ser obtidos pela integração dos componentes.

O método DME trabalha traço-a-traço, consiste em decompor um traço sísmico com banda de frequência larga em vários traços com bandas de frequência estreitas, que são as FMls, sendo que o conteúdo de frequência de cada FMI sobrepõe parcialmente o conteúdo de frequência das FMls vizinhas, o que diferencia o método DME do método de filtragem de frequência. Este método pode ser aplicado nos domínios tempo-espaço $(t-x)$ e frequência-espaço $(f-x)$. As principais inovações conceituais no método DME, é a introdução de FMls baseado em propriedades locais do sinal, o que torna a frequência instantânea significativa; e a introdução da frequência instantânea para conjuntos de dados complexos, que elimina a necessidade de harmônicos espúrios para representar os sinais não-lineares e não-estacionários. A FMI possui transformada de Hilbert bem comportada, a partir do qual as frequências instantâneas podem ser calculadas (Huang e Norden, 1998).
Segundo (Huang e Norden, 1998) uma função em modos intrínsecos é uma função que satisfaz duas condições:

- No conjunto de dados, o número de extremos e o número de cruzamentos por zero, devem ser igual, ou diferir no máximo por um;

- Em qualquer ponto, o valor médio do envelope definido pelo o máximo e o mínimo local é zero.

Essas duas condições asseguram que as FMls serão simétricas com relação à média local e que a frequência instantânea não terá flutuações indesejadas (Ferreira, 2010). As FMls representam modos de diferentes frequências, sendo que para o domínio do tempo, a primeira FMI possui as maiores frequências, enquanto que a última possui as menores frequências. De acordo com a banda de frequência que se deseja preservar, as FMls podem ser subtraídas do dado. Uma importante premissa deste método é que no domínio tempo-espaço $(\mathrm{t}-\mathrm{x})$, a soma de todas FMls geradas da decomposição do sinal, mais o resíduo final, deve compor novamente o sinal inicial.

Dado um traço sísmico de entrada, $x(t)$. O primeiro passo do método DME é calcular os pontos de máximos e mínimos locais para identificar os extremos, fazer a interpolação entre os extremos de máximos e entre os extremos de mínimos, para criar os envelopes dos pontos de máximos e de mínimos, designados de $e_{\sup (t)}$ e $e_{\text {inf }(t)}$ respectivamente (Fig. 1 ).

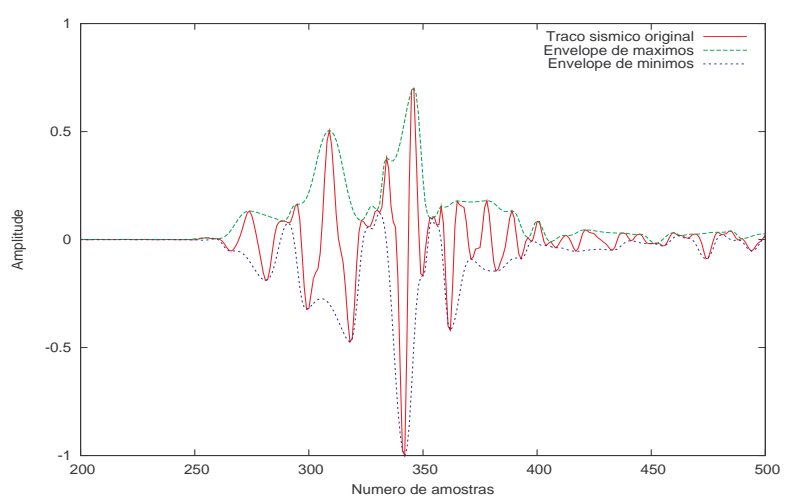

Figura 1: Traço sísmico de entrada em vermelho e os envelopes máximo e mínimo, em verde e azul, respectivamente.

O próximo passo é calcular o envelope médio, que é a média dos envelopes de máximo e mínimo, que é designada como $m_{1}(t)$ (Figs. 2).

$$
m_{1}(t)=\frac{\left[e_{\sup (t)}+e_{i n f(t)}\right]}{2} .
$$

O resultado da diferença entre o dado sísmico de entrada $x(t)$ e a média $m_{1}(t)$ é a primeira componente $h_{1}(t)$, candidata à primeira FMI. 


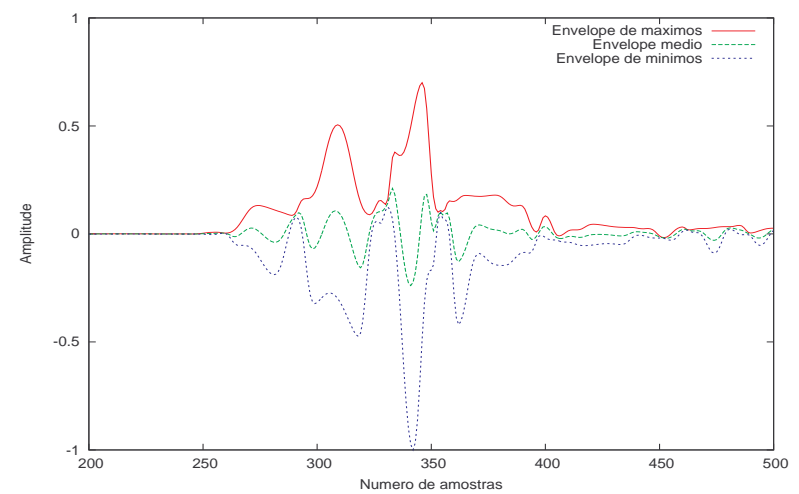

Figura 2: Envelope médio em verde e envelopes dos máximos e mínimos, em vermelho e em azul, respectivamente.

$$
X(t)-m_{1}(t)=h_{1}(t)
$$

Neste caso $h_{1}(t)$ representa um candidato à primeira FMI. Para tornar-se uma FMI, $h_{1}(t)$ deve satisfazer as duas condições proposta por (Huang e Norden, 1998). O sinal $h_{1}(t)$ torna-se o novo dado de entrada e o processo continua até transformar-se em uma FMI.

$$
h_{1}(t)-m_{11}(t)=h_{11}(t),
$$

onde $m_{11}(t)$ representa a nova média do envelope e $h_{11}(t)$ é o novo candidato à FMI, o processo de separação deve se repetir $k$ vezes até que $h_{1 k}(t)$ se torne uma FMI.

$$
h_{1(k-1)}(t)-m_{1 k}(t)=h_{1 k}(t)
$$

Então $h_{1 k}(t)$, será designado como a primeira FMI e

$$
c_{1}(t)=h_{1 k}(t)
$$

Para garantir que a FMI, tenha algum significado físico na modulação da frequência e da amplitude, deve adotar um critério de parada, isto pode ser feito, limitando o tamanho do desvio padrão, SD, calculado entre dois processos de separação consecutivos.

$$
S D=\sum_{t=0}^{M}\left[\frac{\left|h_{1(k-1)}(t)-h_{1 k}(t)\right|^{2}}{h_{1(k-1)}^{2}(t)}\right],
$$

Os valores de referência utilizados para o SD estão normalmente compreendidos entre $0.2 \leq S D \leq 0.3$.

$c_{1}(t)$ (primeira FMI) contém as componentes de alta frequências do sinal. O próximo passo é retirá-la do dado sísmico inicial,

$$
x(t)-c_{1}(t)=r_{1}(t),
$$

$r_{1}(t)$ é o resíduo, que ainda contém as demais componentes de mais baixas frequências do sinal (Fig. 3).

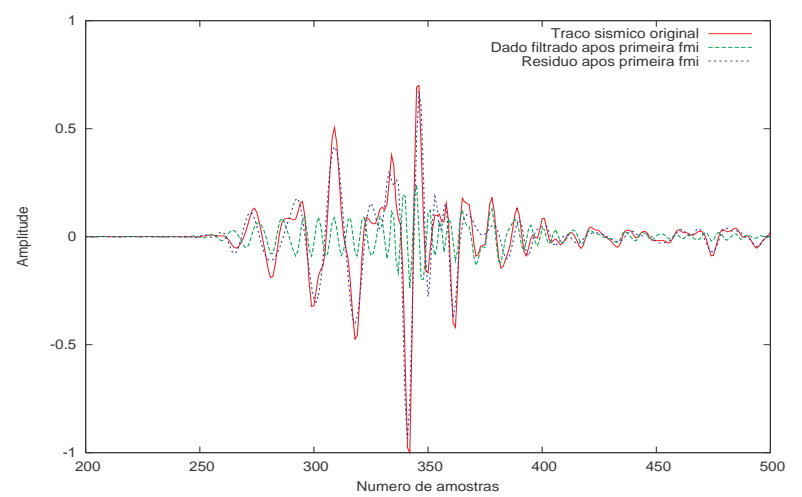

Figura 3: Traço original, $x(t)$ em vermelho, $1^{\circ} \mathrm{FMI}$, $c_{1}(t)$ em verde e o resíduo, $r_{1}(t)$ em azul.

$r_{1}(t)$ será tratado como o novo sinal, e é submetido ao mesmo procedimento descrito anteriormente. Este procedimento deve ser repetido para todos subsequentes $r_{j}(t)$, e o resultado será:

$$
r_{j}(t)-c_{j+1}(t)=r_{j+1}(t)
$$

O processo de separação deve ser interrompido quando atingir o número de FMls desejado, quando a componente, $c_{n}(t)$, ou o resíduo, $r_{n}(t)$, tornam-se tão pequeno, que eles são menores que um valor pre-determinado ou quando o resíduo, $r_{n}(t)$, torna-se uma função monotônica, sem extremos, da qual não seja mais possível extrair qualquer FMI. Mesmo para um sinal com média zero, o resíduo final pode ainda ser diferente de zero e para sinal com alguma tendência, o resíduo final deve representar esta tendência (Huang e Norden, 1998) (fig. 4).

$$
x(t)=\sum_{i=1}^{n} c_{i}(t)+r_{n}(t)
$$

Onde $\sum_{i=1}^{n} c_{i}(t)$ representa a decomposição do sinal de entrada em $n$ FMls e $r_{n}(t)$ representa o resíduo, que pode ser uma tendência média ou uma constante. Para aplicar o método DME, uma média ou uma referência zero não é requerida, pois, somente necessita das localizações dos extremos locais. A referência zero para cada componente será gerada pelo processo de separação de cada FMI. A Figura 5 mostra o resultado da decomposição do sinal sísmico inicial em $8 \mathrm{FMls}$ e o resíduo, que pode representar a tendência do sinal.

A Figura 6 mostra os dados filtrados, após a decomposição da primeira FMI e das oito primeiras FMls respectivamente. 


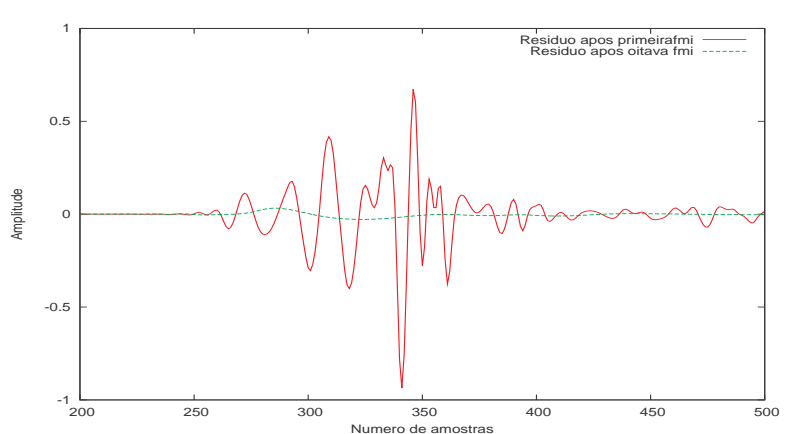

Figura 4: Resíduo após filtragem da $1^{\circ}$ FMI em vermelho e o resíduo após filtragem na $8^{\circ}$ FMI em verde.

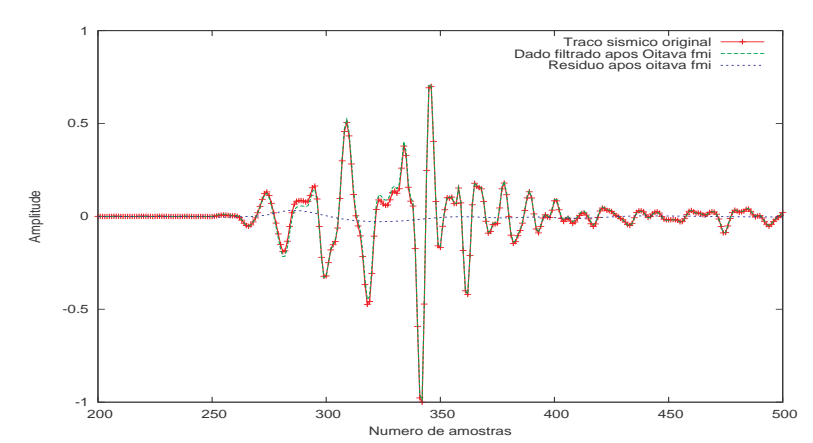

Figura 5: Traco sísmico original em vermelho, traço filtrado obtido com a soma de 8 FMls em verde e o resíduo em azul.

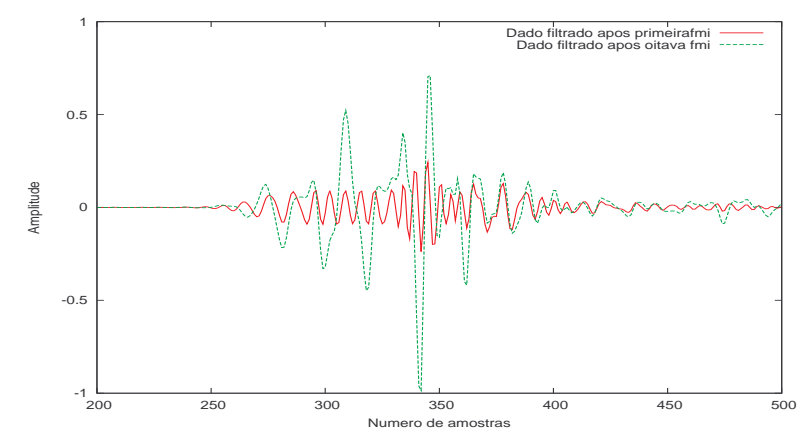

Figura 6: Traço filtrado com a $1^{\circ}$ FMI em vermelho e com as 8 primeiras FMls em verde.
A Figura 7 mostra os espectros de amplitudes do dado original, do dado filtrado e do resíduo após a filtragem da primeira FMI e das 8 primeiras FMls, respectivamente.

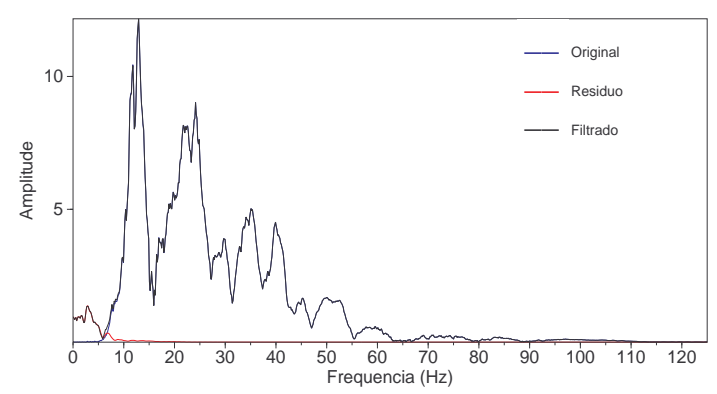

Figura 7: Espectros de amplitude. Sinal original em azul, sinal formado com as 8 primeiras FMls em preto e o resíduo em vermelho.

\section{APLICAÇÃO DO MÉTODO DME NA FILTRA- GEM DE RUÍDO TIPO SWELL NOISE}

O ruído tipo swell noise é muito comum em registro sísmico marítimo quando registrado em condição de mar agitado. A Figura 8 mostra uma família de tiro comum contaminada com o ruído swell noise. A este dado foi aplicado o método DME, decompondo-o em 6 FMls e filtrando-o somando todas as FMls, com o desvio padrão igual a 0.001 . O resultado do dado filtrado pode ser visto na Fig. 9 e o resíduo, que a diferença entre o dado original e o dado filtrado pode visto na Fig. 10.

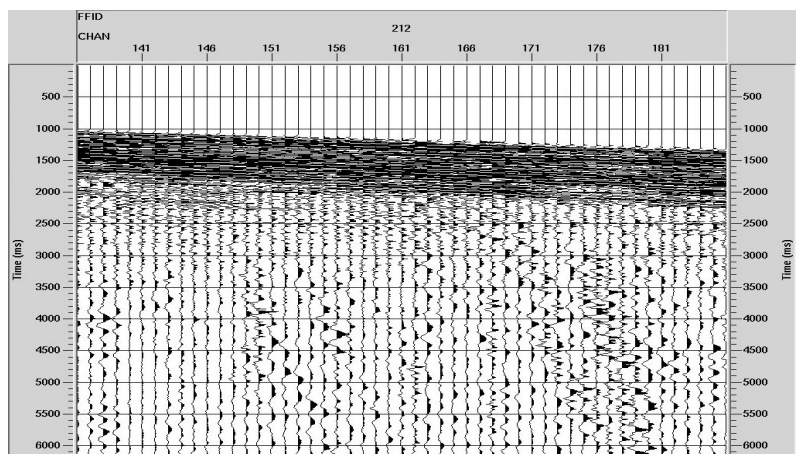

Figura 8: Família de tiro comum, destacando o ruído tipo swell noise.

A Fig. 11 mostra uma família de tiro comum contaminado com o ruído swell noise e seu espectro de amplitude médio, antes da aplicação do método DME. Como podese observar, o ruído possui frequência baixa e amplitude muito alta em torno de $10 \mathrm{HZ}$. A Figura 12 mostra o dado filtrado, composto com a soma das 6 primeiras FMls, e seu espectro de amplitude médio. Observa-se no espectro de amplitude que o ruído foi removido e o sinal ficou preservado. A Fig. 13 mostra o resíduo e seu 


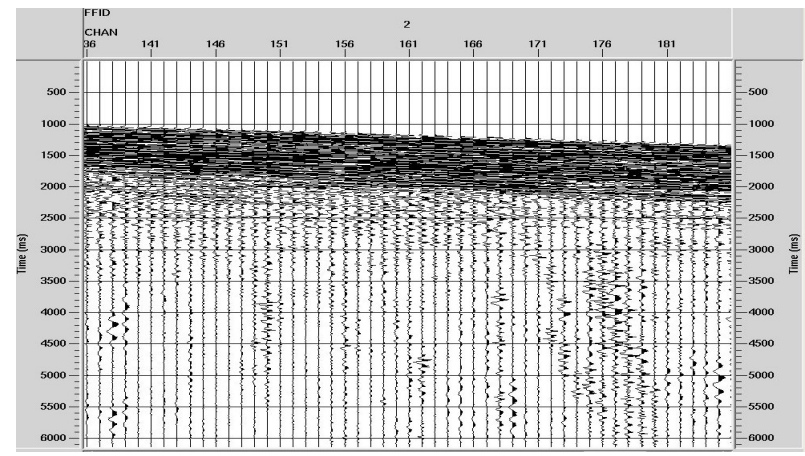

Figura 9: Família de tiro comum, mostrando o dado filtrado após a aplicação da DME.

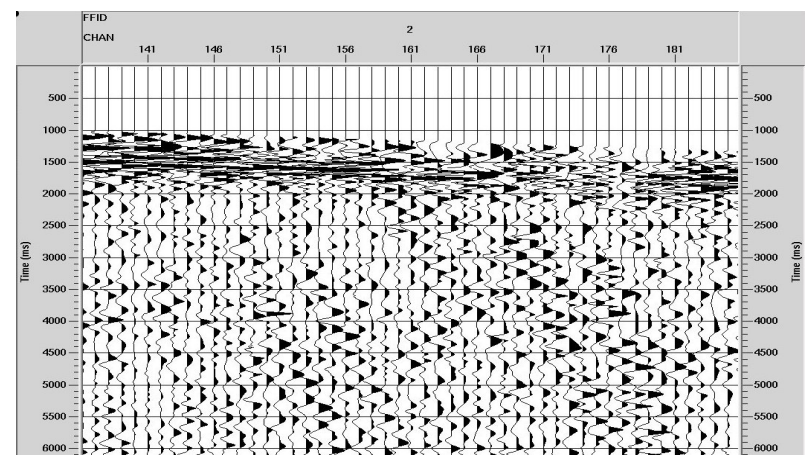

Figura 10: Família de tiro comum, mostrando o resíduo após a aplicação da DME.

espectro de amplitude médio. Observa-se que o resíduo que foi removido é dominado por baixas frequências e alta amplitude em torno da frequência de $10 \mathrm{HZ}$.

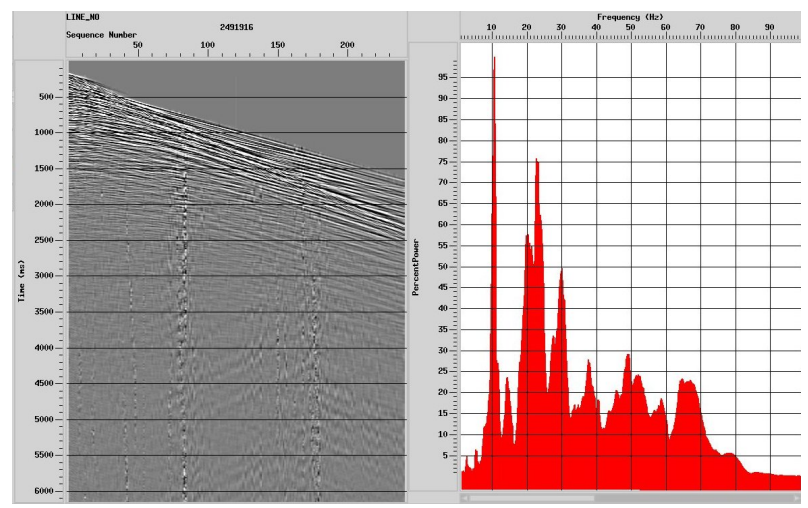

Figura 11: Família de tiro comum do dado original e seu espectro de amplitude médio.

As Figuras 14 a 16 mostram respectivamente, 2 tiros contendo alguns canais contaminados com o ruído, o dado filtrado, e o resíduo após aplicação do método DME, filtrados na $6^{\circ}$ FMI. Podemos observar que a filtragem foi muito eficaz, mostrando o potencial deste método na atenuação e remoção de ruído do tipo swell noise.

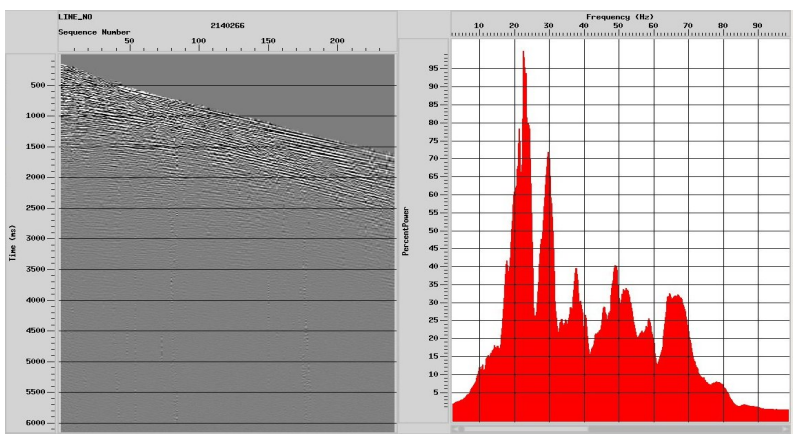

Figura 12: Família de tiro comum do dado filtrado e seu espectro de amplitude médio.

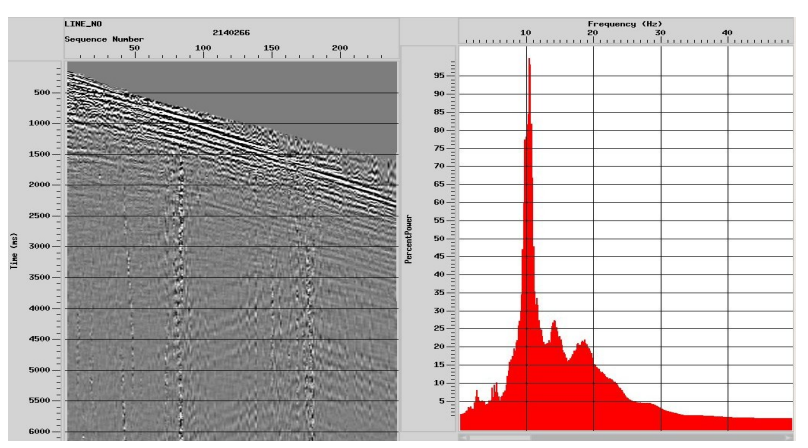

Figura 13: Família de tiro comum do resíduo e seu espectro de amplitude médio.

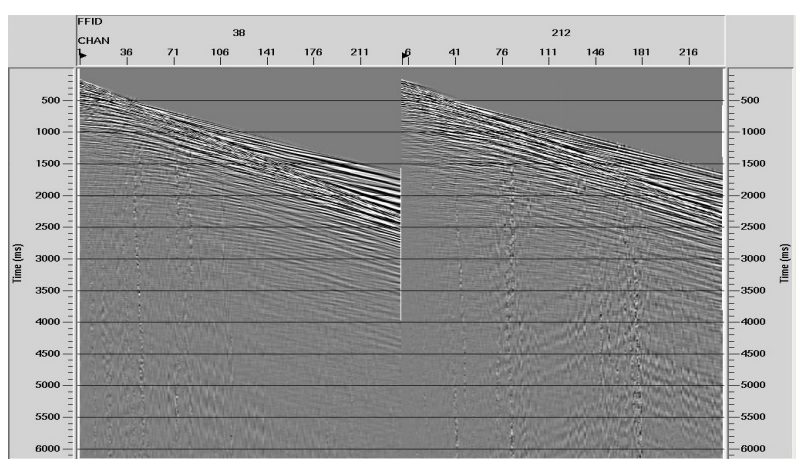

Figura 14: Família de tiro comum destacando o ruído tipo swell noise

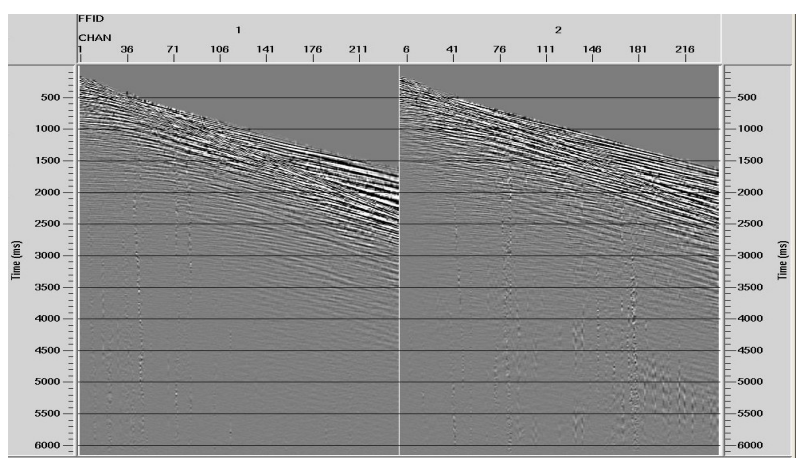

Figura 15: Família de tiro comum mostrando o dado filtrado após a aplicação do método DME. 


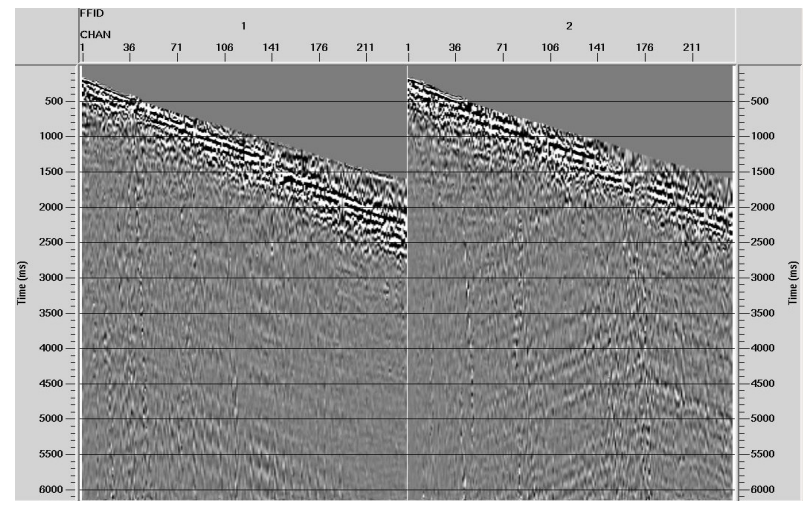

Figura 16: Família de tiro comum destacando o resíduo do swell noise depois de filtrado.

\section{CONCLUSÕES}

O método DME mostrou ser uma ferramenta muito útil na remoção de ruído do tipo swell noise. Este tipo de ruído pode ser removido quando as últimas FMls são excluídas do dado original, isto porque as últimas FMls são dominadas pelas baixas frequência, característica daquele ruído. Ruídos dominados por frequências altas, também podem ser removidos, excluindo-se as primeiras FMls. Adicionalmente, o método DME pode ser aplicado iterativamente, seguindo o procedimento usual. Se o ruído não for totalmente atenuado quando da primeira aplicação, os resultados obtidos podem ser ressubmetidos à filtragem. A eficácia, simplicidade de implementação e eficiência computacional conferem ao método DME algumas vantagens com relação ao método de filtragem em frequência. O método DME por ser um método simples, aplicado no domínio do tempo e por não afetar a amplitude do sinal, possui vantagens em relação aos métodos convencionais utilizado na atenuação do swell noise.

\section{AGRADECIMENTOS}

Á PETROBRAS pelo apoio financeiro e ao INCT-GP/CNPq e CPGG/UFBA pelas facilidades oferecidas para realização deste trabalho.

\section{REFERÊNCIAS}

Alves, F. M., Porsani, M. J., Silva, M. G., e Vasconcelos, G. L., 2011, Filtragem de eventos lineares utilizando os métodos de transformada radial e decomposição em modos empíricos: SEG-XX Congresso Internacional de Geofísica, Rio de Janeiro, Brasil.

Bekara, M., e Van der Baan, M., 2009, Random and coherent noise attenuation by empirical mode decomposition: GEOPHYSICS, 74, V89-V98.

Elboth, T., e Hermansen, D., 2009, Attenuation of noise in marine seismic data: SEG Expanded Abstract, 28, 3312.

Elboth, T., Reif, B. A. P., e Andreassen, O., 2009, Flow and swell noise in marine seismic: Geophysics, 74, Q17-Q25.

Ferreira, L. E. S., Porsani, M. J., e Silva, M. G., 2010, Aplicação do método de decomposição em modos empíricos na atenuação do ruído de rolamento: SBGF- IV Simpósio Brasileiro de Geofísica, Brasília, Brasil.

Ferreira, L. E. S., 2010, Aplicação do métdo de decomposição em modos empíricos na atenuação do ruído de rolamento em dados sísmicos: Ph.D. thesis, Universidade Federal da Bahia, Salvador, Bahia, Brasil.

Flandrin, P., Rilling, G., e Gonçalvés, P., 2004, Empirical mode decomposition as a filter bank: IEEE SIGNAL PROCESSING LETTER, 11, 112-114.

Huang, E., e Norden, E., 1998, The empirical mode decomposition and the hilbert spectrum for nonlinear and non-stationary time series analyses: Royal Society London, pages 454:903-995.

Meignen, S., e Perrier, V., 2007, A new formulation for empirical mode decomposition based on constrained optimization:

Zegadi, A., Kleir, K., e Zegadi, E., 2009, Coherent and radom noise attenuattion using the intrinsic timescale decomposition: SEG-Internation Exposition and Annual Meeting, Houston, Texas, USA, pages 33223326. 